\title{
Early treatment with cyclosporin in serpiginous choroidopathy maintains remission and good visual outcome
}

\author{
A A Q Araujo, A P Wells, A D Dick, J V Forrester
}

\begin{abstract}
Aims-To describe management and clinical outcomes of serpiginous choroidopathy treated primarily with cyclosporin at a tertiary uveitis referral centre

Methods-A case series of 14 eyes of seven patients with serpiginous choroidopathy with follow up ranging from 1.3 to 13 years is described. All patients had fundus lesions consistent with serpiginous choroidopathy, were investigated for systemic disease, had fluorescein angiography, and were treated with combined immunosuppressive therapy including cyclosporin.

Results-No patients suffered significant loss of acuity after starting systemic immunosuppression with cyclosporin as the primary agent. All but one patient achieved remission and were able to stop medications with no recurrences in the follow up period. Side effects from cyclosporin were well tolerated and there were no serious complications from immunosuppression.

Conclusions-Cyclosporin is a safe and effective option with which to manage serpiginous choroidopathy. Significantly, adequate immunosuppression can result in clinical remission and cessation of therapy in some patients.

(Br f Ophthalmol 2000;84:979-982)
\end{abstract}

Serpiginous choroidopathy is a disease of sufficient rarity that most ophthalmologists outside tertiary referral centres will have little contact with it, and even among experts there is debate about what is the most appropriate treatment. There is inflammatory chorioretinopathy characterised by geographic areas of choroidal atrophy in both eyes. These typically occur at the disc and spread in a helicoid pattern along the major vascular arcades towards the macula, although there may be variations including a macular type. ${ }^{12}$ Recurrences are common and manifest as yellow-grey extensions at the level of the choriocapillaris, contiguous or as satellites to existing areas of chorioretinal atrophy. In approximately one third of cases there is vitreous activity. ${ }^{3}$ Histology of active lesions ${ }^{4}$ shows a lymphocytic infiltrate, most prominent in the choriocapillaris at the edge of the lesions, and extending into the choroid and retinal pigment epithelium. Choroidal atrophy and mild pigmentary change is prominent in previously affected areas. Visual disability may result directly from retinal lesions affecting the central macula, or from secondary choroidal neovascularisation ${ }^{5}{ }^{6}$ resulting from disruption of the Bruch's membrane-retinal pigment epithelium (RPE) complex. Because the pathogenesis of serpiginous choroidopathy involves lymphocyte activity, ${ }^{4}$ many clinicians elect to treat with immunosuppression. ${ }^{37-10}$ The range of systemic regimens reported is from observation only to triple therapy with corticosteroids, azathioprine, and cyclosporin $\mathrm{A},{ }^{10}$ the success of which remains conflicting.

\section{Patients and methods}

PATIENTS

Case series of patients with serpiginous choroidopathy were retrieved from the Aberdeen Uveitis Database. All patients recorded in the database as "serpiginous" over a 10 year period were classified according to International Uveitis Study Group guidelines ${ }^{11}$ resulting in seven patients (14 eyes) with definite serpiginous choroidopathy and adequate follow up data. The International Uveitis Study Group provides criteria to exclude other diseases which may masquerade as serpiginous choroidopathy. ${ }^{11}$

CLINICAL CHARACTERISTICS AT PRESENTATION Ages ranged from 24 to 44 years, mean 34 years, and there were five men and two women (Table 1). Four patients had one eye with vision less than $6 / 36$, and there were seven eyes with visual acuity of $6 / 9$ or better (Fig 1). All patients had bilateral disease at diagnosis. The range of disease durations before definitive diagnosis was from 5 days to 72 months. No patients had coexisting ocular disease.

INVESTIGATIONS

All patients had full ophthalmic examination including best corrected visual acuity and fun-

Serpiginous choroidopathy-case definition

- Bilateral geographic areas of choroidal atrophy and scarring, centred on optic disc, with characteristic pattern of spread along vascular arcades towards macula

- Quiet eye with quiet anterior chamber

- With or without active choroidal inflammatory lesions, appearing as yellow-grey areas contiguous with, or as satellites to, existing areas of chorioretinal atrophy

- With or without mild posterior vitritis 
Table 1 Patient characteristics

\begin{tabular}{llllllllc}
\hline Case & $\begin{array}{l}\text { Age/sex/ } \\
\text { duration }\end{array}$ & Eye & Initial VA & Final VA & $\begin{array}{l}\text { Years of } \\
\text { follow up }\end{array}$ & Rx now & Relapse & $\begin{array}{c}\text { Activity } \\
\text { free years }\end{array}$ \\
\hline 1 & $35 / \mathrm{M} / 36$ & L:R & $6 / 12: 6 / 9$ & $6 / 12: 6 / 9$ & 8.5 & Yes & Off $\mathrm{Rx}$, at 6 years & 2.5 \\
2 & $44 / \mathrm{M} / 72$ & L:R & $6 / 7.5: 2 / 60$ & $6 / 6: 2 / 60$ & 6 & No & No & 5 \\
3 & $29 / \mathrm{F} / 5$ days & L:R & $6 / 60: 6 / 12$ & $6 / 36: 6 / 7.5$ & 4.1 & No & No & 10 \\
4 & $34 / \mathrm{M} / 7$ & L:R & $6 / 5: 6 / 5$ & $6 / 6: 6 / 12(\mathrm{~N} 5)$ & 5.6 & Yes & Multiple & 1 \\
5 & 29/M/1 & L:R & $6 / 5: 6 / 5$ & $6 / 6: 6 / 6$ & 3 & Yes & No & 2.5 \\
6 & 24/F/1 & L:R & $6 / 6: 6 / 36$ & $6 / 5: 6 / 36$ & 1.3 & Yes & No & 1 \\
7 & $44 / \mathrm{M} / 2$ & L:R & $6 / 6: 6 / 60$ & $6 / 6: 6 / 60$ & 2.4 & Yes & No & 2
\end{tabular}

Duration = time between onset of disease and initiation of immunosuppression. Initial VA = Snellen visual acuity at initiation of immunosuppression. Final VA = Snellen visual acuity at last visit. Years of follow up = years of follow up since initiation of immunosuppression. Rx now = "Yes" if patient is still taking immunosuppressive drug, "No" if not. Relapse = "No" if there was no activity of disease at any follow up since initiation of immunosuppression. Activity free years = years of no detectable disease activity after initiation of immunosuppression.

*Patient had been immunosuppressed for 6 years and on a low dose of cyclosporin at time of relapse. After increased dose, patient has been free of activity since. $†$ Patient required unusually aggressive immunosuppression.

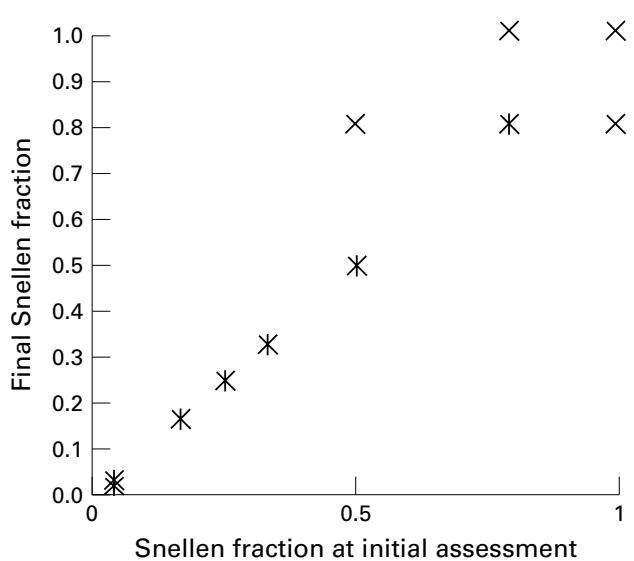

Figure 1 Visual acuity at initial assessment versus final visual acuity for all patients. The single patient whose distance vision decreased because of mild cataract retained N5 reading vision.

dus biomicroscopy. Colour fundus photography and fluorescein angiography were performed in all cases. All patients were screened to exclude systemic causes of posterior uveitis with Mantoux test, syphilis serology, chest $x$ ray, and serum angiotensin converting enzyme.

\section{INDICATIONS FOR THERAPY AND DECISION TO} TREAT

The decision to treat with cyclosporin A was made if there was detectable disease activity in either eye at initial assessment. Disease activity was diagnosed if there was typical creamy white lesions at the retinal pigment epithelial level with early hypofluorescence, and late staining or leakage on fluorescein angiography (Fig 2). ${ }^{1}$

\section{IMMUNOSUPPRESSION}

All patients were treated initially with a combination of systemic cyclosporin A and prednisolone. Two patients were additionally treated with azathioprine, and one (case 4) also had tacrolimus and mycophenolate mofetil.

ONGOING CARE

The immunosuppressive regimen was induction with cyclosporin A at 3-5 $\mathrm{mg} / \mathrm{kg} /$ day (Table 2) and prednisolone at a variable dosage depending on disease control at first assessment at Aberdeen, ranging from $5 \mathrm{mg} /$ day to 100 $\mathrm{mg} /$ day. The cyclosporin dose was decreased if the serum creatinine rose by more that $30 \%$ above baseline levels or if other side effects occurred. In addition to this treatment, two patients were given supplementary immunosuppression: cases 2 and 4 both had azathioprine, and case 4 who had very refractory disease also required tacrolimus and mycophenolate mofetil.

All patients were followed up at 1-4 week intervals while the disease was active, and 3 monthly when inactive. Treatment outcome was assessed by VA and funduscopy. Blood pressure, weight, and serum creatinine, liver enzyme, and electrolyte levels were checked and complete blood cell count and urinalysis performed at each visit. Cyclosporin levels were obtained to monitor patient compliance and potential toxicity. The glomerular filtration rate was checked at baseline and once a year, unless the serum creatinine rose more than $30 \%$ above baseline values and failed to return to baseline with reduction of the cyclosporin dose.

\section{Results}

Of the 14 eyes, 10 had the same final visual acuity as at first assessment, one eye worsened, and three improved (Table 1). Five of the seven patients had no relapses at an average follow up of 4.1 years, and two of these five patients have been medication free for 5 and 10 years. Currently the average duration of disease free interval is 3.6 years, range $1-10$ years. Only one eye lost distance visual acuity due to lens changes, and this eye retained N5 near vision (see Fig 1).

Two patients have had recurrence of activity over the follow up period. Patient 1 had a recurrence approximately 2 years after he stopped medication prematurely due to poor compliance. Patient 4 had refractory inflammation which did not respond to cyclosporin A and high dose oral corticosteroids and required more aggressive immunosuppression.

Side effects of cyclosporin A were notably inconsequential (Table 3). Two patients had troublesome malaise or other systemic symptoms, magnesium levels rarely required supplementation, and with one exception creatinine levels did not stay elevated for more than 2 months. No patients has had a permanently elevated creatinine or other serious side effect. 

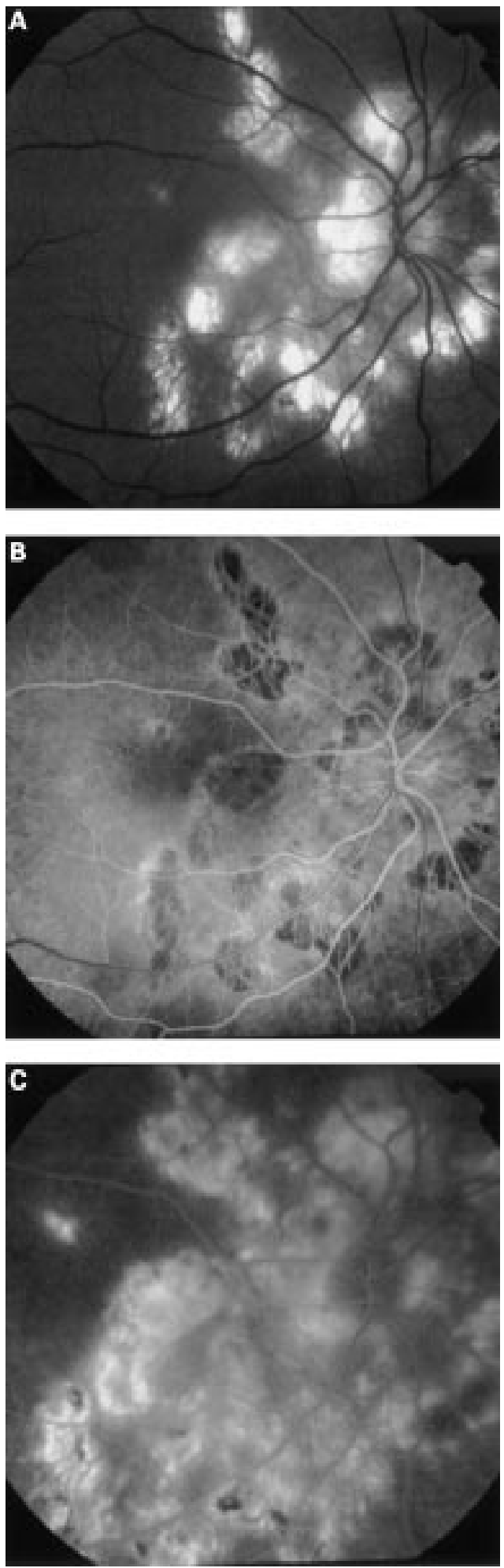

Figure 2 Fluorescein angiogram (right eye, patient 4). Note the typical geographic areas of choroidal atrophy radiating from the disc and spreading towards the macula. There is a new, small area of disease activity approximately 1 disc diameter above the fovea visible on the red-free photograph $(A)$, which in the angiogram is characterised by early hypofluorescence $(B)$ and late hyperfluorescence $(C)$.

\section{Discussion}

This paper shows the effectiveness of an immunosuppressive regimen based on cyclosporin $\mathrm{A}$ in the management of serpiginous chorioretinopathy. Secchi and colleagues ${ }^{7}$ also described good outcomes where nine of 14 eyes showed significant recovery of visual acuity with cyclosporin therapy, although other reports $^{89}$ indicated poor therapeutic results. Cyclosporin monotherapy was ineffective, ${ }^{8}$ but when triple agent immunosuppression was used with azathioprine, cyclosporin, and prednisone $^{10}$ the inflammatory response was controlled. In these conflicting reports immunosuppression was started only when sight was threatened or when the second eye was affected, which may deny the best potential outcome for the patient.

The spectrum of alternative immunosuppressive therapies for serpiginous chorioretinopathy ranges from monotherapy with corticosteroids or other agents alone to triple therapy with multiple agents. ${ }^{10}$ Because it is so rare there is no prospective controlled trial comparing treatments, only case series similar to this one, thus there is no firm evidence for efficacy of any regimen. In many of these studies, ${ }^{7-10}$ the decision to treat was based on existing or imminent visual loss, and poor outcomes have been reported. Although there is no evidence for herpesvirus group involvement in the pathogenesis, there are unpublished reports of aciclovir speeding recovery in a few patients. However, serpiginous choroidopathy has a course characterised by exacerbations and remissions, so treatments should be judged on their ability to prevent visual loss over several years.

One concern with respect to the natural history of serpiginous choroidopathy is the unpredictability of relapses where if active lesions extend to the fovea irreversible visual loss will result. Visual prognosis is generally thought to be poor in this disease. Macular involvement occurs with consequent decreased visual acuity in up to $88 \%$ of patients ${ }^{39}$ and approximately $50 \%$ could be expected to have recurrence in 5 years. ${ }^{8}$ On the basis of the present study we suggest that the timing for treatment should be at presentation with active choroiditis, so that disease control can be maintained on acceptable low doses of immunosuppression, such as cyclosporin A. The ultimate aim of immunosuppression is to prevent progressive active lesions involving the macula by suppressing the inflammatory response. This rationale is based on the most widely accepted pathogenesis, that of non-infectious $\mathrm{T}$ cell mediated inflammatory response ${ }^{12} 13$ which is supported clinically by histopathological demonstration of Iymphocytic choroidal infiltrates. ${ }^{4}$ Additionally, significantly raised factor VIII-Von Willebrand factor has been reported in eight patients with serpiginous choroidopathy, implicating a vasoocclusive mechanism. ${ }^{14}$

We show promising outcomes in all of our seven patients including long term remission while maintaining excellent acuity. The majority have not had relapse of their disease after an average 4.4 years of follow up and only one particularly refractory case had a recurrence while still using cyclosporin A. Importantly, we did not have as large a proportion of visual acuity improvement as in other studies but we did elect to treat on the basis of disease activity 
Table 2 Medications

\begin{tabular}{llllll}
\hline Case & Pred/d $(\mathrm{mg})$ & Duration pred & $\begin{array}{l}\text { CsA }(\mu \mathrm{g} / \mathrm{l}) \\
\text { repres }\end{array}$ & $\begin{array}{l}\text { Duration } \\
\text { CsA (years) }\end{array}$ & Systemic treatment \\
\hline 1 & $1.25^{\star}$ & 3 years, 2 years & 600 & $4.5,2 \dagger$ & Ster, CsA \\
2 & $12.5^{\star}$ & 4 years & 300 & 3.5 & Ster, CsA, Aza \\
3 & 20 & 1 month & 300 & 2.5 & Ster, CsA \\
4 & 100 & 3 months, 1 month, 1 month & 500 & $5 \dagger$ & Ster, CsA, Aza, FK-506, myco, aciclovir \\
5 & Nil & Nil & 200 & $3 \dagger$ & CsA alone \\
6 & 40 & 1 & 250 & $1.3 \dagger$ & Ster, CsA \\
7 & $20^{\star}$ & 2.4 & 200 & $2.4 \dagger$ & Ster, CsA \\
\hline
\end{tabular}

Pred/d = initial dose of oral prednisolone per day. Duration pred = duration of prednisolone treatment (note weaned to smaller doses for long term use). CsA repres = representative blood level of cyclosporin A during treatment. Duration CsA = years of cyclosporin use; CsA = cyclosporin A; Aza = azathioprine; Ster = corticosteroids; FK-506 = tacrolimus; Myco = mycophenylate mofetil. ${ }^{\star}$ These patients were initiated on prednisolone at other centres. †These patients are still taking cyclosporin.

Table 3 Side effects of cyclosporin

\begin{tabular}{llllllll}
\hline Case & Malaise & Cramps & Mg & Incr BP & Other & Creat incr & $\begin{array}{l}\text { Final creat } \\
(\mu m o l / l)\end{array}$ \\
\hline 1 & $\mathrm{~N}$ & $\mathrm{~N}$ & 1.25 & $\mathrm{~N}$ & $\mathrm{~N}$ & $\mathrm{~N}$ & 82 \\
2 & $\mathrm{~N}$ & $\mathrm{~N}$ & $\mathrm{NA}$ & $\mathrm{N}$ & $\mathrm{N}$ & $\mathrm{N}$ & 97 \\
3 & $\mathrm{~N}$ & $\mathrm{~N}$ & 0.74 & $\mathrm{~N}$ & $\mathrm{I}$ & Y-2 months & 77 \\
4 & $\mathrm{Y}$ & $\mathrm{N}$ & 0.85 & $\mathrm{~N}$ & $\mathrm{G}, \mathrm{L}$ & Y-single test & 80 \\
5 & $\mathrm{~N}$ & $\mathrm{~N}$ & 0.78 & $\mathrm{~N}$ & $\mathrm{~N}$ & Y-1 year & 106 \\
6 & $\mathrm{~N}$ & $\mathrm{~N}$ & 0.71 & $\mathrm{~N}$ & $\mathrm{~N}$ & Y-2 months & 93 \\
7 & $\mathrm{~N}$ & 0.83 & $\mathrm{~N}$ & $\mathrm{~N}$ & $\mathrm{~N}$ & 92 \\
\hline
\end{tabular}

Malaise $=$ any recorded systemic symptom (tiredness, fatigue, nausea, vomiting, temperature intolerance) recorded in case notes, regardless of whether this necessitated change in treatment. Cramps $=$ any muscle cramps recorded in case notes. Incr $\mathrm{BP}=$ rise in blood pressure sufficient to require change in treatment. Other $=$ any other adverse effects recorded in case notes, regardless of whether this necessitated change in treatment: $\mathrm{I}=$ secondary infection (infectious keratitis), $\mathrm{G}=$ gingival hyperplasia, $\mathrm{L}=$ lymphopenia (transient in this case). Creat incr $=$ raised level of plasma creatinine above $120 \mu \mathrm{mol} / \mathrm{l} ; \mathrm{N}=$ nil, $\mathrm{Y}=$ yes with duration specified.

rather that foveal involvement, so eight of our eyes had $6 / 9$ or better visual acuity at the initiation of treatment.

Despite the good outcome, the data must still be interpreted with respect to the rarity of the disease, the retrospective open study design, and the lack of controls. It is noteworthy that none of these patients had the complication of choroidal neovascularisation (CNV), given that this occurs in $20-26 \%$ of cases in other series. ${ }^{35}$ Part of this decreased incidence and thus improved visual prognosis may be related to immunosuppression, which has been used to successfully treat CNV associated with endogenous posterior uveitis. ${ }^{15}$

There were few significant side effects to cyclosporin A in this cohort. No patient had constitutional symptoms severe enough to necessitate ceasing cyclosporin A and surprisingly few reported any significant problems with malaise, fatigue, anorexia, nausea, hirsutism, or gingival hyperplasia. Cyclosporin A appears to be an effective and safe drug for preventing progression and inducing remission in patients with serpiginous choroiditis. We would therefore recommend immunosuppressive therapy with cyclosporin A in management of serpiginous choroiditis, and prompt initiation of treatment, before involvement of central vision in either eye.
1 Schatz H, Maumenee A, Patz A. Geographic helicoid peripapillary choroidopathy: clinical presentation and fluorescein angiographic findings. Trans Am Acad Ophthalmol Otolaryngol 1974;78:747-61.

2 Mansour AM, Jampol LM, Packo KH, et al. Macular serpiginous choroiditis. Retina 1988;8 125-31.

3 Weiss H, Annesly WJ, Shields JA. The clinical course of serpiginous choroidopathy. Am $\mathcal{F}$ Ophthalmol 1979;87 133-42.

4 Wu JS, Lewis H, Fine SL, et al. Clinicopathologic findings in a patient with serpiginous choroidopathy and treated choroidal neovascularisation. Retina 1989;9:292-301.

5 Jampol LM, Orth D, Daily MJ. Subretinal neovascularisation with geographic (serpiginous) choroiditis. $A m$ f tion with geographic (se

6 Laatikainen L, Erkkila H. Subretinal and disc neovascularisation in serpiginous choroiditis. Br f Ophthalmol 1982; 66:326-31.

7 Secchi AG, Tognon MS, Maselli C. Cyclosporin A in the treatment of serpiginous choroiditis. Int Ophthalmol 1990; 14:395-9.

8 Laatikainen L, Tarkkanen A. Failure of cyclosporin A in serpiginous choroiditis. $f$ Ocul Ther Surg 1984;3:280-3.

9 Nussenblatt RB, Palestine AG. Uveitis: fundamentals and clinical practice. Chicago: Year Book Medical, 1989:313.

10 Hooper PL, Kaplan HJ. Triple agent immunosuppression in serpiginous choroiditis. Ophthalmology 1991,98:944-51.

11 Bloch-Michel E, Nussenblatt RB. International Uveitis Study Group recommendations for the evaluation of intaocular inflammatory disease. Am f Ophthalmol 1987; 103:234-5.

12 Forrester JV. Uveitis: pathogenesis. Lancet 1991;338:1498501.

13 Caspi RR, Roberge FG, Chan CC, et al. A new model of autoimmune disease. Expenmental autoimmune uveoretinitis induced in mice with two different retinal antigens. retinitis induced in mice with

14 King DG, Grizzard WS, Sever RJ, et al. Serpiginous choroidopathy associated with elevated factor VIII- Von Willebrand factor antigen. Retina 1990;10:97-101

15 Dees C, Arnold JJ, Forrester JV, et al. Immunosuppressive treatment of choroidal neovascularisation associated with endogenous posterior uveitis. Arch Ophthalmol 1998;116: 1456-61 